\title{
Eye movement contingent display control in studying reading
}

\author{
GEORGE W. McCONKIE, DAVID ZOLA, GARY S. WOLVERTON, \\ and DAVID D. BURNS \\ Department of Education, Cornell University, Ithaca, New York 14853
}

\begin{abstract}
A computer system has been developed that permits experimental control over a CRT display contingent upon characteristics of the viewer's eye movements. The display can be changed during specific saccadic eye movements or fixations. Uses of such a system for studying reading are described. The paper reviews hardware and software considerations in developing such a system.
\end{abstract}

In normal visual tasks such as examining objects, looking for some pattern, or reading, the eye makes a series of fixations at the rate of about $4 / \mathrm{sec}$. With such frequent change of eye position, it is difficult to investigate in detail the perceptual and cognitive processes taking place during and across fixations. For instance, what information is obtained on particular fixations, what information is combined across fixations, what decisions are involved in controlling when and where the eye is sent for the next fixation, and so on? As a result, two general approaches have been taken to the study of such questions. One approach is to investigate the events occurring during a single fixation by simulating a fixation with a tachistoscopic presentation. The tachistoscope provides the ability to control the stimulus sequence in ways that permit the investigation of a number of interesting psychological questions. Control is exercised over the sequence of still images that are placed upon the retina, their position with respect to each other, and their timing with respect to some external event (typically, the press of a button or the tick of a clock). The nature of the tachistoscope has limited this research in several ways. First, it has been difficult to provide more than three alternative images, which limits the types of stimulus patterns that can be produced. This problem has been overcome to a great extent with computerized displays for stimuli that can be easily represented with characters or simple line drawings (Taylor, Klitzke, \& Massaro, 1978) or by multiframe projectors if a constant sequence is desired (Forster, 1970; Potter, 1976). A more critical limitation has been on the types of tasks that can be studied with a tachistoscope. Typically, the task has involved a single fixation, with

Preparation of this paper was supported in part by Grant MH24241 from the National Institute of Mental Health. The paper was presented at the National Conference on the Use of On-Line Computers in Psychology, Washington, D.C., in November 1977. Requests for reprints should be addressed to the senior author at the Department of Education, Cornell University, Ithaca, New York 14853. the series of images occurring during the fixation and some report following. Thus, the normal dynamics involved in reading or in visually examining a scene are lost. It is likely that the perceptual and cognitive events occurring in a task that involves grasping information during a single fixation will be quite different from the events occurring during a particular fixation embedded in the entire reading or viewing task. So, the results from tachistoscopic studies may not generalize to the tasks that we really wish to understand.

The other approach to research has been to keep the task as natural as possible, while giving up most of the stimulus control that the tachistoscope makes possible. Thus, the person may be shown a picture or text, his eye movements may then be recorded, he may be asked to make some judgment during the task so response time can be recorded, or he may be presented with test items following the task to see what he retained from it. The lack of stimulus control has made it difficult to investigate many questions about perceptual and cognitive processing with this approach, although it has the strength that the findings obtained do relate to the task we wish to understand.

The development of the laboratory computer, with equipment for monitoring eye movements and for exercising dynamic control over visual displays, makes it possible to establish a research environment that combines the strengths of the two approaches. For several years, we have been developing a computer system that is capable of monitoring people's eye movements as they read text displayed on a cathode-ray tube (CRT) and that can make changes in the text based on eye movement information. We can change the display during an eye movement so the text pattern on one fixation is not the same as it was on the previous fixation, or we can change the display at certain times during a fixation. The computer system, called EYECON, permits EYE movement CONtingent control over the display as the person visually examines it. The system is briefly described later in the present 
paper. The primary purpose of the paper is to discuss some of the hardware and software considerations involved in developing such a system. Previous descriptions of such systems can be found in reports by Reder (1973) and by McConkie and Rayner (Note 1).

\section{SOME EXAMPLES OF THIS TYPE OF RESEARCH}

A few examples of studies that have been conducted using the computer techniques described above may indicate some of the uses for this type of technology.

McConkie and Zola (Note 2) investigated whether strictly visual characteristics of the text stimuli are carried from one fixation to the next by having subjects read text printed in alternating case format (e.g., AlTeRnAtInG cAsE) on a CRT, and then changing the case of every letter during certain saccades. Although some aspect of the shape of every letter was changed from one fixation to the next, subjects were unaware of the change and their eye movements were unaffected.

McConkie and Zola (Note 3) shifted the entire line of text two letter positions $(2 / 3 \mathrm{deg})$ to the right or left during a saccade as subjects read from it and found that, while subjects were not consciously aware of the shift, their eye movement patterns were affected.

Rayner (1975) investigated how far into the periphery subjects acquire visual information during a fixation by changing one word to another as the subject made a saccade directed to that word while reading. His data suggested that subjects acquire visual information from a word only if their eyes are centered no more than 12 letter positions to the left of it, and that they make semantic interpretations of a word only if their eyes are centered no more than 6 letter positions to the left of it.

McConkie and Rayner (1975) and O'Regan (1975) changed the display on each fixation as subjects read in a manner that left normal text in a region a certain number of letter positions to the left and right of the center of vision, with letters in the peripheral visual regions being replaced by other letters (e.g., Xs). These studies provided information about what aspects of stimuli are used from different distances into the periphery during fixations in reading. McConkie and Rayner (1976) found a great asymmetry in the reader's perceptual span with information being used from a very limited region to the left of the center of vision.

Such studies as these are only possible through technology that permits the stimulus pattern to be controlled on the basis of eye movement information. They have the advantage that, in every case, the subject was engaged in a task of reading to understand a passage or short paragraph and was later tested for comprehension. In many instances, subjects were not aware that experimental manipulations were taking place during the study.
There are two problems in making eye movement contingent display changes: (1) developing a way to make the desired display changes, and (2) developing a way to cause the changes to occur at the appropriate time.

\section{TYPES OF DISPLAY CHANGES}

Both hardware and software decisions are heavily influenced by the types of display changes needed in the planned research. It is assumed here that the stimuli are to be computer generated on a CRT or plasma display, although the issues raised can be extended to other types of displays as well (television, transparencies, etc.).

The first distinction is whether the display change is to be discrete or continuous. This distinction is not as important as it might first appear, since we know from the cinema and television industries, as well as from psychological research, that even continuous changes in a display can be simulated by a sequence of static images. Thus for the present purposes, all display changes are viewed as a series of static images. The basic differences in display changes, then, are in the number of images required (the number of "frames"), the speed with which these must replace each other, and the density of locations at which the stimulus can be changed (and perhaps the number of alternatives at each of these locations; e.g., the number of colors or levels of intensity). If the researcher only wishes to replace letters in a text with other letters at the same locations on the display, an alphanumericoriented CRT capable of displaying a fixed set of characters at fixed locations may be quite sufficient, given that the changes can be made fast enough. If relatively continuous changes are desired (moving a line of text smoothly across the CRT, gradually distorting one letter until it comes to look more and more like another, etc.), a greater density of addressable stimulus points is needed, such as is available in highdensity point-plotting or vector-generating video systems. Thus, it is important to correctly anticipate the types of stimulus changes needed and to obtain equipment capable of speeds and densities required for the research.

In developing the software, the important considerations are the number and complexity of the frames, since these influence whether alternative frames can be prepared in advance and stored in high-speed memory or must be generated as needed by algorithm. The first of these is the stored alternatives approach; the second is the generated alternatives approach. The stored alternatives approach is typically fastest, especially if the alternatives can be stored in core memory, but it is more costly in terms of storage space. The generated alternatives approach is conservative of memory but requires more computer time. 
Within the stored alternatives approach, efficiency is sometimes gained by storing alternatives for only part of the total stimulus pattern. Thus, if most of a display will remain the same and only part will change, alternative subpictures for that part can be stored and selected as needed. In reading research, this might consist of storing altematives for a particular word or line in the text.

Since a line of text requires relatively little storage space (36 16-bit words for a 70-character line plus carriage return and line feed), alternative versions of a line can be stored in core memory and control switched from one to another to produce many of the display changes needed in the study of reading. A number of interesting display changes can be produced in this manner. Shifting the location of a line to left or right can be accomplished by having alternative lines containing the same text but with different numbers of spaces at the left. If the lines are identical except for one word, displaying a different line alternative changes the word in that text location. If one alternative consists of all Xs, and it is displayed $100 \mathrm{msec}$ after a fixation begins, the line of text originally displayed during that fixation will be masked. Displaying a line of part or all spaces removes part or all of the line. A surprising number of display changes can be produced by simply displaying alternative lines of text when desired, a fact long recognized by researchers working with the tachistoscope.

When the number of needed frames becomes too large to permit their storage in high-speed memory, the generated alternatives approach must be taken. This is particularly likely to occur when the appearance of smooth motion is required, or when there is a large number of alternative displays that might be needed depending upon characteristics of the subject's eye movements. The complexity of the stimulus generation algorithm needed can vary widely, but for many purposes the algorithms can remain quite simple. McConkie and Rayner $(1975,1976)$ provide examples of this approach. In their studies, at the beginning of each of the reader's fixations, the computer identified the letter in the text lying closest to the center of vision and replaced all letters more than a certain distance to the left and right with other letters. This left a "window" of normal text of a particular width at the region being fixated by the subject and changed the text pattern in peripheral visual areas in the specific ways needed for the research. Figure 1 presents a simple method for generating this type of stimulus pattern once the eye position has been identified. Two alternative versions of the line are stored in the computer. When the eye position is identified, the display is constructed by taking parts from each of these alternatives to form the new line. With some equipment configurations (to be discussed later), this can be accomplished easily by simply inserting
Ine Alternatives stored in the computer:

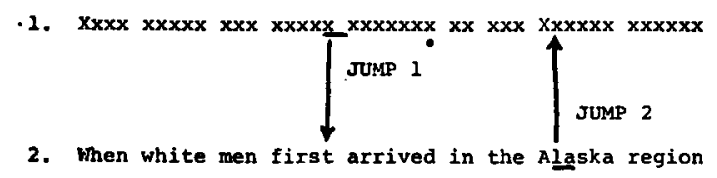

Line appearing on the CRT during the indicated fixation:

Xxxx $x \times x \times x$ xxx xxxxt arrived in the Axxxxx $x \times x \times x \times$

Figure 1. Constructing a window display. The computer receives eye position information and determines that the letter marked with a circle lies at the center of vision. It then replaces the underlined letters in Line 1 with a JUMP instruction that transfers control to Line 2 . A second JUMP is inserted into Line 2, which returns control to Line 1 . The computer then begins the display at the beginning of Line 1, producing the new line shown at the bottom of the figure.

jump instructions at the appropriate locations in the two line alternatives themselves. Again, a large number of display changes can be produced using this simple algorithm, as shown in Figure 2.

While these comments hardly begin to describe the rich potential computer graphics provides for psychological research, they do illustrate that many interesting display manipulations needed for psychological studies can be produced relatively easily and quickly, and without necessarily writing a new program for each one. Rather, each algorithm can be developed to produce a family of display changes.

\section{DECIDING WHEN TO MAKE THE DISPLAY CHANGE}

Just as there are many types of display changes that

$$
\begin{aligned}
& \text { Standard line: } \\
& \text { When white men first arrived in the Alaska region } \\
& \text { Alternative lines: } \\
& \text { 1. ..............t arrived in t........... } \\
& \text { 3. Wher xklfe ncm tinet arrived in tke Risahs neqlom } \\
& \text { 4. John Smith had heard Wrrived in the Aister crying } \\
& \text { 5. ..................................... }
\end{aligned}
$$

Figure 2. Five examples of displays that can be created for a specific fixation. Example 1 deletes peripheral visual information. In Example 2, letters in the periphery are replaced by visually similar letters. In Example 3, letters in the foveal region are removed and reading occurs from peripheral information alone. In Example 4, text in the periphery is taken from a different passage than that in central vision. In Example 5, an $x$ is always placed at the location where a subject fixates. This can be useful in subjectively testing the accuracy of the system in identifying eye position. (The $\uparrow$ indicates the letter on which the eye is centered for that fixation.) 
can be produced, there are also many ways to define when a change is to occur. Typically, the experimenter will wish to specify whether a display change is to be made during a saccade or during a fixation, and during which saccade or fixation. Also, he may wish to specify when during the fixation or saccade the change is to occur. In general, the decision of when to produce the change may involve information about: (1) whether the eye is moving or still; (2) if moving, the direction of movement and saccade rate characteristics (present velocity, peak velocity and when it occurred, etc.); (3) how long since the eye entered this saccade or fixation; (4) how many fixations or saccades have occurred thus far on the line or page (perhaps broken down by forward and regressive movements); (5) the location or predicted location of the eye; and (6) some history consisting of a sequence of these elements. For a given experiment, the computer program must identify the aspects of eye behavior from this list that are needed to decide when a change is to occur, match this information against some pattern defined by the experimenter, and be able to recover from instances when a match has begun but failed to be completed. For many purposes, the pattern to be matched will be very simple (i.e., make a change on the second, fourth, and sixth saccade of every line as soon as the eye is detected to be moving). For other purposes, the pattern can become much more complex.
There are many timing considerations involved in the question of whether a display change can in fact be made at the time desired by the experimenter. These considerations are the focus of the remainder of this section. In particular, the discussion will deal with the problem of changing the display during a saccadic movement, since this is a capability often desired for reading research and involves most of the timing considerations encountered under other circumstances.

\section{Some Characteristics of Saccadic Movement in Reading}

Figure 3 shows the horizontal component of one forward eye movement of about 10 letter positions in length (3-1/3 deg) taken from the data of a college student reading a passage from a CRT. As with most data presented here, eye position was sampled every millisecond. This saccade required about $42 \mathrm{msec}$ to complete. It has been our experience that the shortest saccades typically found in reading, making an excursion of one letter position ( $1 / 3 \mathrm{deg})$ or less, take about $18 \mathrm{msec}$. Saccades of average length (eight to nine letter positions, or about $3 \mathrm{deg}$ ) take $30.50 \mathrm{msec}$. Eye movements that return the eye to the next line (return sweeps) require about $90 \mathrm{msec}$. There is a strong, curvilinear, monotonic relation between the lengths of saccades and the time they require to complete.

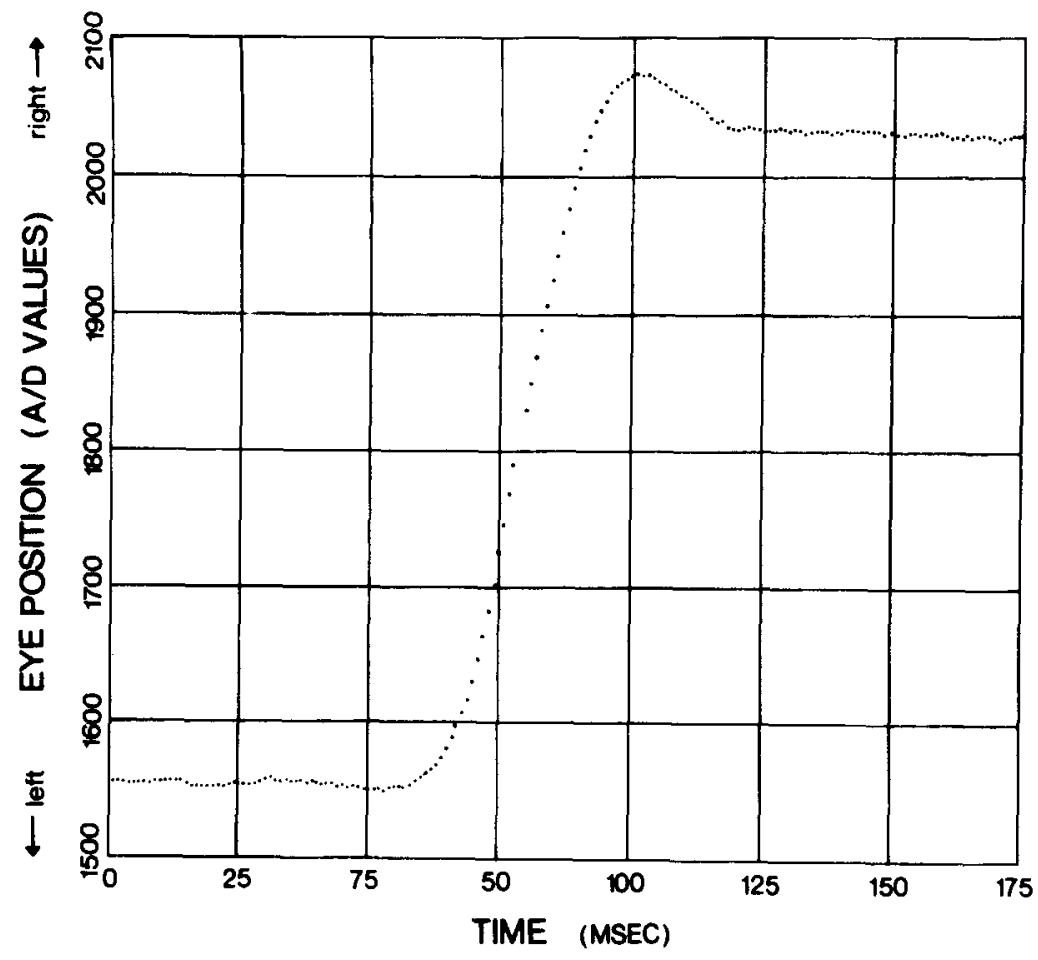

Figure 3. Data from a typical rightward saccade in reading of about $3.3 \mathrm{deg}$ excursion (about 10 letter positions). Eye position was sampled every millisecond. The vertical axis represents eye position on a scale in which an eye movement of 1 letter position ( $1 / 3 \mathrm{deg}$ ) corresponds to a change of about 50 values. 
If the experimenter wishes to complete a display change during the shortest saccades in reading, this must be done within $18 \mathrm{msec}$ of the time the eye begins to move. Longer eye movements provide more time.

Sometimes the experimenter will wish to make display changes only on saccades of a certain length (for instance, only if they will take the eye at least $1 \mathrm{deg})$. This presents the added problem of predicting the length of a saccade while it is in progress. As Figure 3 indicates, the eye typically speeds up, reaches a peak velocity around midway in the saccade, and then gradually slows. The data at the beginning of the saccade do not reveal how far the eye is likely to travel. The first indicator of saccade length is the point at which the increase in velocity begins to drop. A second indicator is the point at which peak velocity is reached. Finally, the peak velocity itself is monotonically related to the length of the saccade. From such information, a prediction can be generated about the likely length of the saccade. The point in the saccade at which peak velocity is reached differs somewhat between subjects, but is fairly constant within subjects, at least for saccades of lengths typically found in reading. Thus, it is possible to determine from a subject's eye movement record how far through the saccade his velocity tends to peak and have the computer apply that constant to estimate the length of a saccade while it is in progress, once the point of peak velocity has been obtained. A decision of whether to make the display change can then be based on that prediction. Of course, this leaves less than half of the duration of the saccade for the change to be produced. When less precision is needed in the prediction, as when it is desired to make a change only if the eye is going to travel more than a given distance (e.g., no display changes are to be made during saccades of less than $1 \mathrm{deg}$ ), it is possible to identify maximum peak velocities for saccades that are to be excluded and then make the display change only if the velocity of the eye exceeds these values.

The important point to be made here is that timing considerations are critical in this type of research. Saccades are short at best; when display changes are to be made only on saccades selected according to their predicted length, the time is constrained even more. Since it is possible to predict the length of a saccade while it is in progress, one can predict during a saccade where the eye will stop for the next fixation, typically within one or two letter positions of its actual location. Thus, display changes can be produced during a saccade that are contingent upon where the eye will be centered for the next fixation.

There are a number of steps that must occur during these short periods: detecting the initiation of a saccadic eye movement, deciding whether a change should occur during this saccade, obtaining the information needed to set up the new display, setting up the display instructions, and, finally, producing the new stimulus pattern on the face of the display device.

\section{Detecting an Eye Movement}

With saccades lasting such a short time, it is critical to detect the onset of a saccade as soon as possible after the eye begins to move, in order to provide the maximum time for later activities before the saccade ends. There are three factors that can greatly affect how early in a saccade the eye movement can be detected: sampling rate, noise in the signal, and lag in the propagation of the signal.

Figure 4 shows the effect that a slow sampling rate can have on detecting the onset of a saccade. The bottom curve presents eye movement data sampled every millisecond; the top curve shows how the same data would have appeared if eye position had been sampled only every $20 \mathrm{msec}$ (50 samples/sec). The initiation of an eye movement can be detected much earlier at the higher sampling rate.

Detection of a movement is typically done by setting some threshold, and when the difference in eye position between successive samples exceeds this threshold, a saccade is said to have started. Of course, it is possible to sample the eye position after a saccade has begun without the eye movement being detected; the amount of movement may not yet have been sufficient to exceed the threshold. In general, one can assume that if the eye position is being sampled every $\mathrm{N}$ msec, in the worst case a saccade will not be detected until $\mathrm{N}$ plus at least 3 or $4 \mathrm{msec}$ after the eye begins to move. At the 20 -msec sampling rate shown in Figure 4, it is quite possible to fail to detect a saccade taking $20 \mathrm{msec}$ until after it has been completed. An example of such an instance is shown in the figure. Even sampling eye position every $5 \mathrm{msec}$ can delay the detection of a saccade to at least 8 or $9 \mathrm{msec}$ after it has begun.

A second undesirable characteristic of relatively slow sampling rates is the variability involved in the point during the saccade at which it is detected. How early a saccade is detected depends on how long it is initiated following the point at which an eye position sample is taken. With a 20 -msec sampling rate, while at times a saccade may not be detected until 24 msec after its initiation, at other times it may be detected within 3 or $4 \mathrm{msec}$. With slow sampling rates, there is considerable variability concerning when during the saccade its onset is detected, and hence how much time remains for making a display change and when during the saccade the change occurs.

We have found it useful to sample eye position every millisecond, comparing the data values with those obtained $4 \mathrm{msec}$ previously. In this way we can usually detect the onset of a saccade within 3 or $4 \mathrm{msec}$ of the time the signal begins to rise or fall.

Noise in the eye position signal can also delay the detection of a saccade, as shown in Figure 5. Passing 


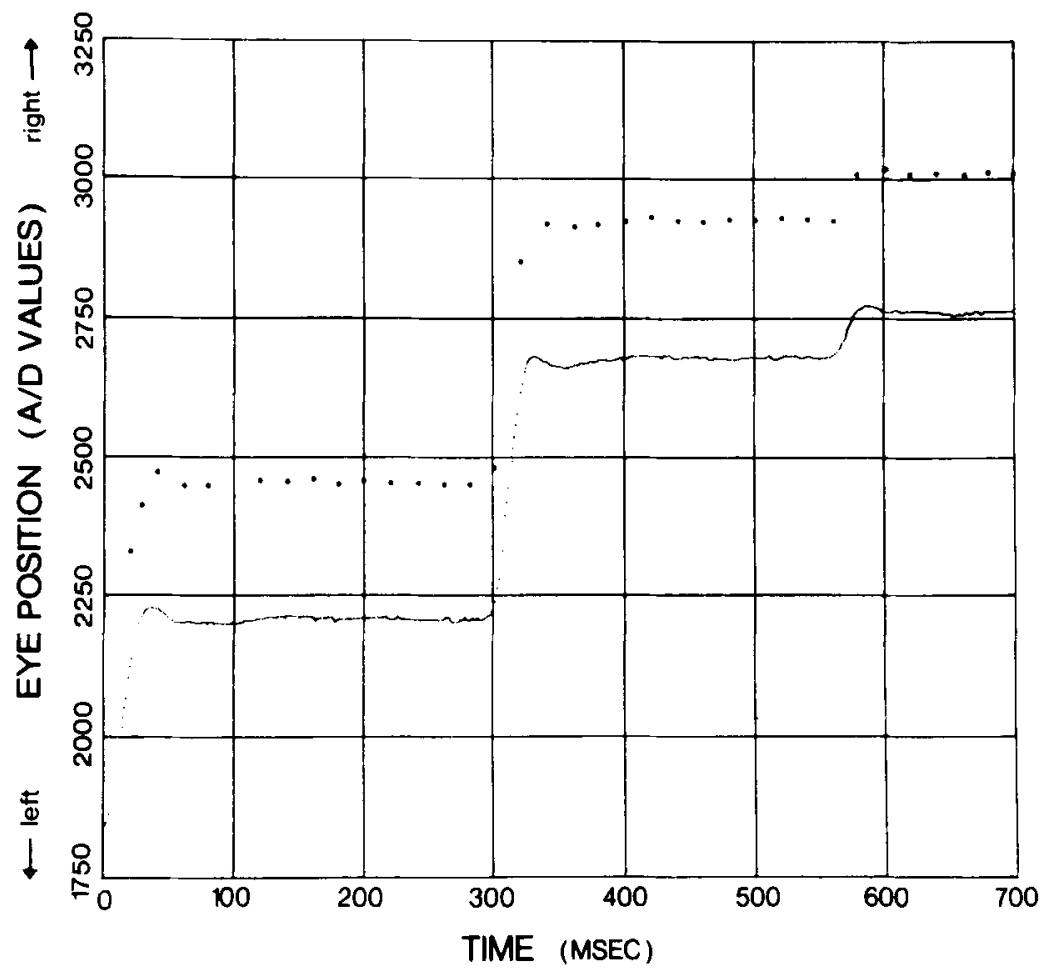

Figure 4. Effects of slow sampling rate. The bottom curve shows eye movement data with eye position sampled every millisecond. The top curve shows what the data would have looked like (somewhat elevated) if eye position has been sampled only every $20 \mathrm{msec}$.

from left to right on the two versions of the data presented, the computer can reliably identify the beginning of a saccadic movement earlier in the cleaner signal. With noisy data, the movement threshold must be set higher to avoid false positive errors. This means that, on the average, the eye must reach higher velocities before its movement is detected; thus the detection occurs only later in the saccade. As Figure 5 shows, a relatively small amount of noise can greatly decrease the accuracy of identifying when a saccade begins and ends.

Finally, the propagation lag of the signal in the eye movement equipment itself can delay the detection of a saccade. With TV monitoring equipment, for instance, it takes $16 \mathrm{msec}$ to make a scan of the eye, process the information, and make an eye position indicator available to the computer. At the end of the scan, information obtained at the beginning is already $16 \mathrm{msec}$ old.

Electrooptical methods of monitoring eye movements (limbus reflection techniques, etc.) require a certain amount of filtering of the electrical signal; this filtering introduces delays of varying amounts. The worst case is shown by the Biometrics (now Narco-Bio) Type SG eye movement monitor, which provides the opportunity to engage a filter for reducing the effects of 60-cycle intensity changes in the ambient lighting. The filter delays the signal about $25 \mathrm{msec}$, so for many saccades the signal the computer receives does not rise until after the movements are complete. With the filter switched off, the lag is around $3 \mathrm{msec}$.

When mechanical movement is required in eye

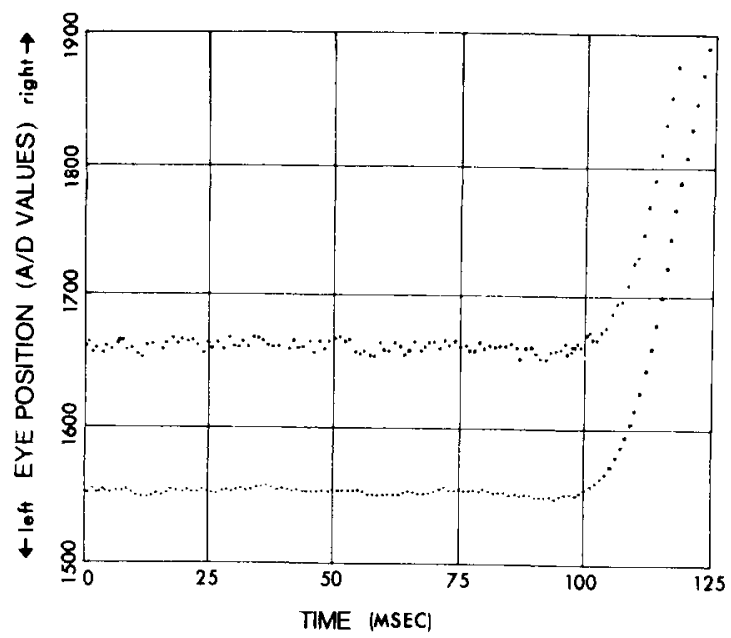

Figure 5. Effects of noise in the data signal. The bottom curve shows eye movement data with low noise level. The top curve shows the same data, displaced upward, with random noise added. The maximum noise added corresponds in amount to an eye movement of $\pm 2.5 \mathrm{~min}$ of visual angle (slightly more than .10 of a letter position). Even this small amount of noise greatly reduces accuracy of detecting the point at which a saccade begins. 
movement monitoring equipment, another source of lag is introduced. This is characteristic of the Standard Research Institute Double Purkinje Image eye movement monitor (Cornsweet \& Crane, 1973), where servomechanisms move mirrors to track the eye. However, the design of this equipment has reportedly reduced such lag to $3 \mathrm{msec}$. Equipment that increases the field of view of eye movement monitoring equipment by moving mirrors to track head movement, and so on, introduces varying amounts of lag time, depending on the characteristics of the equipment.

In summary, with existing equipment capable of being interfaced with the computer, the time at which the onset of a saccade can be detected ranges from about 5 or $6 \mathrm{msec}$ after the eye begins to move (with sampling eye position every millisecond, a 2 - to 3 -msec propagation lag in the equipment and a very low noise level) to over $30 \mathrm{msec}$. In the worst cases, only the longest saccades are detected before they are completed.

There are two other undesirable effects of slow sampling rates and high noise levels. First, it becomes difficult to distinguish between saccadic eye movements and other slower movements of the eye (see comments of Levy-Schoen \& O'Regan, Note 4, as an example). Also, many small saccades can be missed entirely. These problems largely (but not entirely) disappear under more optimal conditions. Second, since slow sampling rates and high noise levels make it difficult to identify when saccades begin and end, they reduce the accuracy of measurements of fixation or saccade durations, which are often critical dependent variables in eye movement research.

\section{Deciding Whether This is a Saccade on Which a Change Should Occur}

How much time is consumed in the attempt to decide whether a change should occur during a given saccade (or possibly, which change should occur) depends on two factors: the complexity of the algorithm involved (together with the speed of the processor) and when the information needed to make the decision becomes available from the saccade itself. In many instances the time involved is very short. The experimenter need only know that a saccade has begun, and perhaps its direction (left or right) and its number (i.e., changes occur only during the third forward saccade on this line).

As explained earlier, a considerable delay is introduced if the decision involves the predicted length of the saccade. With such prediction algorithms, the decision concerning the display change often cannot be made until after the eye has passed its point of peak velocity, thus leaving relatively little time to carry out the remaining activities (about half the total saccade time minus the delays induced by the propagation lag, etc.). These decisions require a fast sampling rate and low noise level to yield accurate information about the point of peak velocity, actual velocity attained, and so on.

In summary, the delay created in deciding whether a change should be made is variable, depending on the nature of the decision required and the type of information needed to make the decision.

\section{Obtaining Information Needed \\ to Produce the Change}

Even when the decision to make a change is simple, the nature of the change itself may require information that only becomes available later in the saccade. This problem is likely to arise in cases where the nature of the display is contingent upon the next fixation's predicted eye position. The display cannot then be constructed or selected until a prediction has been generated. Typically, information needed for the prediction is not available until the latter half of the saccade. How complex the prediction algorithm must be depends on the linearity of the eye position signal and the degree of accuracy required. All of these factors, together with the speed of the processor, influence how early the needed information can be made available and the next step can be taken. Even with optimal equipment, it is possible to have only $24 \mathrm{msec}$ remaining after the predicted eye position has become available before a short saccade is finished (peak velocity is identified $11 \mathrm{msec}$ into the saccade, there is a $3-\mathrm{msec}$ propagation lag, $1 \mathrm{msec}$ is required for generating the prediction, and the total saccade duration is only $18 \mathrm{msec}$ ).

\section{Making the Change in the Display Instructions}

So far, only the preliminaries to changing the display have been discussed. The process of making the change involves (1) the computation required to create the display instructions in memory and to transmit this information to the display device, and (2) the time required to realize the display change on the screen.

Computation and transmission time depends on several factors: the complexity of the calculations required, the speed of the computer, and the speed with which the resulting changes can be passed to the display device. As indicated earlier, many interesting changes can be created by simply replacing one line with another; others can be created by combining information from several alternative lines to create some composite. In these cases, the computation required is minimal. Thus, the primary consideration is the rate at which information can be passed to the display device.

There are three types of relationship between the computer and the display device, and the arrangement chosen can influence timing considerations considerably. In the first case, the computer controls the display device directly. That is, it not only sets up the list of information to be displayed (the display list), but also 
continually passes pieces of information to the display device in order for the image to be constantly refreshed. With this type of system, other activities of the computer are being interrupted many times during each millisecond in order to pass to the display the information it needs. This, of course, considerably reduces the amount of computing time available to other activites, and hence slows the calculations involved in other stages of the process being described. On the other hand, once the change is to be made and the new display list has been created or selected, the change can be implemented without delay. The computer simply passes the new information instead of the old. In the second case, the computer creates the display list, then transmits this information to the memory of the display device. From then until the next display change is required, the CPU is relieved of any responsibility of tending the display. The display device takes care of the constant refreshing of the image. This system frees the CPU from the constant display-tending responsibilities, leaving its entire time available for the other calculations necessary. The desirabillity of this approach depends on the speed with which the display information can be passed to the display device. This varies from machine to machine. These machines also vary in whether the entire display list must be transmitted in order to produce a change, or whether only the parts that are different must be sent. This transmission time ranges from less than $1 \mathrm{msec}$ to $16 \mathrm{msec}$ (where transmission of information must be in phase with the raster scan of the display device) or even longer. The amount of this delay must be added to the total delay time for producing the display change.

The third case is the most desirable, but also the most expensive. It involves having two processors, both with access to the same core memory. One is the computer's central processing unit (CPU) and the other is a display processor (DPU) that has the sole responsibility for controlling the display on the CRT. With this arrangement, the CPU can set up the display instructions in core memory and simply point the DPU to where they are located. The DPU takes care of refreshing the display, with no further attention from the CPU. The time required is simply that needed to create the display list: There is no separate transmission time, and the CPU is not burdened with display responsibilities. Thus it is completely free for other computation. With this arrangement, complex display changes can be created and the display process initiated within a fraction of a millisecond.

\section{Producing the Change of the Display on the CRT}

Once the computer creates the new list of display instructions and transmits it to the display device, if necessary, there can still be a considerable time lag before the display is present on the screen. Here, the primary concern is whether the CRT is refreshed by a raster scan, or whether individual points or lines can be written in any specified order. Commercially available raster-scan CRTs typically refresh the image every $16 \mathrm{msec}$ in order to produce a flicker-free image. It is not possible to interfere with the scanning process other than to turn it on or off; that is, it is not possible to stop a scan midway and initiate a new scan. When a change is desired, it is necessary to wait until the scanning process reaches the point on the screen where the new display is to occur. This will be $8 \mathrm{msec}$ on the average and a full $16 \mathrm{msec}$ in the worst case. The delay has two effects: It adds considerably to the time required to make a display change, and it adds variability to when a display change will actually occur. This is not under the control of the computer. With some CRTs it is possible to provide the computer with a signal when a scan begins, so it is possible, after the fact, to know how great the delay was before a change appeared on the screen.

Most changes in simple displays can be realized in much less time on CRTs having random-access pointplotting capabilities. In work with text, this is particularly true if the equipment includes a hardware character generator. With such equipment, refresh cycles can be considerably shorter than $16 \mathrm{msec}$, and it is possible to interrupt a cycle and begin the display of some new part of the image immediately. For instance, we use a Digital Equipment Corporation (DEC) VT-11 display system with a hardware character generator. Even though the character gesierator is relatively slow for present technology, a line of 71 letters can be displayed in $2.05 \mathrm{msec}$. With a single line of text, changes in the display can be realized on the screen in about $2 \mathrm{msec}$ without breaking the refresh cycle. Shorter times are possible if the refresh cycle is interrupted, but this may result in some irregularity in the perceived brightness of different parts of the display. We have not yet explored the effects of this alternative.

The decay characteristics of the phosphor used on the CRT are critical for this research. The image should certainly not persist longer than the refresh period; crisp display changes can be produced by using phosphors that largely decay in $1 \mathrm{msec}$ or less. However, the phosphor adopted as a standard on a piece of equipment is usually not critical in a purchase decision, since companies will usually supply the equipment with a different phosphor in response to special request. If not, the manufacturer of the tube will usually sell duplicate tubes with a different phosphor that can be installed in the equipment.

While the preceding discussion has been oriented to CRT displays, there are alternatives that may be much more appropriate for given purposes. For example, plasma displays having the necessary characteristics for this type of research are likely to become available shortly. Their desirable characteristics include very fast onset and offset times and a constant image that does 
not need refreshing. At the present time, however, the commercially available plasma displays receive data very slowly, and the transmission time required is too great to permit use in the type of research described here. This is not a limitation of the plasma tubes themselves, but only of the presently available equipment designs.

\section{Conclusions}

It should be clear that, while it is possible to make display changes during a saccadic eye movement, there are many equipment and software considerations that come into play if it is to be done effectively. With presently available technology, in the worst case (TV-based eye movement monitor with 16-msec sampling rate, fairly high noise, $10 \mathrm{msec}$ required for creating the display change and transmitting it to the display device, and a full refresh cycle of the raster-scan display device before the change is actually made on the screen), it is quite possible for the change to be realized in visible form only $55 \mathrm{msec}$ or more after the eye begins its movement. This is likely to be $25-30 \mathrm{msec}$ after the next fixation has begun. On the other hand, in the best case, the change can be reliably produced about 8-9 msec following saccade onset, and it even becomes possible to control the point during the saccade at which the change is made. Equipment decisions are critical to anyone wishing to do research of this type. Clearly, the greatest time wasters are pieces of equipment that operate by raster scan, whether as eye movement monitoring systems or as display devices.

In order to determine whether a particular equipment configuration will be adequate for conducting a specific experiment, two tasks are required. First, the researcher must identify the nature of the timing requirements of the study if it is to be conducted successfully. Second, he must attempt to estimate, from manufacturers' equipment descriptions together with educated guesses concerning computing time involved, the amount of time that will be required in each of the steps discussed above. Only in this way is it possible to make an intelligent decision about whether the equipment being considered is adequate for the research desired.

Finally, this discussion has assumed that the display changes need to occur during the period of the saccade. It is possible that with saccadic suppression and masking processes occurring, changes can occur shortly after the end of the saccade without changing the data patterns obtained. This is, of course, an empirical question that can only be studied by having a system capable of making changes either during or immediately after the saccade. If there is a period of time following the onset of a fixation during which display change can occur without disrupting the normal visual processes, the conduct of research will be made much easier, and researchers will operate under fewer constraints. However, "saccadic suppression" is somewhat misleading. The description refers, not to a complete blocking of visual input during (and immediately preceding and following) a saccade, but only to a somewhat raised threshold (Matin, 1974). It is likely that the intensity of the displays used for most psychological research lies above this threshold, and hence is capable of being registered by the visual system, even during the saccadic suppression period.

\section{DISPLAY CHANGES DURING FIXATIONS}

For some research questions, it is necessary to make changes in the display during a fixation, rather than during a saccade. On the one hand, there are fewer constraints in this type of research, since the shortest fixations seldom fall below $100 \mathrm{msec}$ (although occasional fixations are seen in the $70-\mathrm{msec}$ range during reading). Thus, more time is available for the processes involved in stimulus control. On the other hand, many of the concerns here are the same as those in making changes during a saccade. Usually, the experimenter wishes to produce the change a certain time following the onset of the fixation. This requires accuracy in identifying when a saccade terminates, which is subject to all the same types of problems involved in determining when a saccade begins, as discussed earlier. Also, there are all the problems associated with producing the new display on the CRT when desired. In fact, if it is desired to make the display change soon after a fixation begins (say, 20-30 msec), most of the problems involved in producing such a change during a saccade will be encountered.

There is one additional problem involved in making display changes during a fixation. The actual point at which a saccade can be said to terminate (and hence, a fixation begin) is buried in a phenomenon sometimes referred to as overshoot. At the end of a saccade, there is often a hump in the data, as seen in Figure 3. This hump is probably associated with the eye and its parts settling into position after the force applied by the ocular muscles to create saccadic movement. This overshoot is exaggerated even further in data collected by the Stanford Research Institute eye tracker, which monitors Purkinje images reflected from the lens. Apparently, the lens either slips somewhat or is distorted in shape as a result of the forces applied during the eye movement, and a considerable settling process occurs at the beginning of the fixation. The result is that final information about where the eye is centered is not available until $30 \mathrm{msec}$ or so into the fixation, and in fact, the point at which the saccade can be said to have terminated remains somewhat in doubt. A similar problem occurs with particularly long saccades such as return sweeps. Such movements are often followed by periods of slow drift of the eye, which are probably vergence adjustments (Alpern, 1971). Again, the exact point of the saccade termination is not clear, and the information concerning where the eye will 
finally be centered is not available at the outset of the fixation. But these problems are not great and, usually, regularities can be observed and compensated for in the computer algorithms.

For certain purposes, it would be desirable to be able to produce display changes a certain time prior to the onset of a saccade. While it may be possible at some future time to monitor the activity of a part of the brain that signals the future occurrence of a saccade a constant time prior to its initiation, that possibility is not available to us now. At present, the experimenter can only produce changes at given times following the saccade onset, and then later, group the data according to how long the change occurred prior to the end of the fixation. This is a questionable practice, because the duration of the fixation may be affected by the stimulus change.

\section{AN EXISTING SYSTEM FOR EYE MOVEMENT CONTINGENT DISPLAY CHANGES}

EYECON is the computer system that we have developed for research involving eye movement contingent control of the display.

\section{Hardware and Environmental Characteristics}

EYECON is built around a DEC PDP-11/40 computer, with two RK05 disk drives, $28 \mathrm{~K}$ words of core memory, a laboratory peripheral system having a realtime clock and eight 12-bit A/D channels, and a DEC VT-11 CRT (with hardware character generator) driven by a display processor. The display unit is a pointplotting scope having alphanumeric and vectorgenerating modes. It has $\mathrm{P}-4$ phosphor, which is white in color and decays to $1 \%$ of the original intensity in 500 microsec.

A Biometrics Model SG eye movement monitoring system of the limbus reflection type (Young \& Sheena, 1975 ) is used to track the eye. This equipment has been rather extensively modified in order to reduce noise and drift in the signal and to increase the accuracy of the clock. The sensors are mounted on micromanipulators to facilitate adjusting the equipment to the proper eye position. A bite bar reduces head movement, and the laboratory is provided with a dc lighting system to reduce light intensity variations.

\section{Software Characteristics}

The software for EYECON occupies $18 \mathrm{~K}$ words of core memory, runs under the RT-11 monitor, and consists of four subsystems, all written in the PDP-11 assembly language: parameter definition system, calibration system, data collection system, and display control system. A separate collection of programs is used to visually examine and to reduce the data.

Parameter definition system. An attempt has been made to develop a program having a great deal of flexibility, and which is easily modifiable for conducting experiments involving eye movement contingent display changes of various types. Thus, there are many parameters that can be used to indicate the options to be taken for a specific study. In order to facilitate the conducting of studies, the program has a macrofacility by which it is able to accept strings of commands from a file that sets the parameters for a particular experiment. These files can be of two types: study files, which specify the parameters that will be held constant for a given experiment (the rate at which data are to be taken, the nature of the calibration period), and session files, which further specify the parameters to be used for a particular data-collection session (the order in which passages are to be read, the nature of the manipulations to be made, etc.). Thus, once a study file has been prepared, the number of parameters with which the experimenter must be concerned in running a study is greatly reduced. If a session file has been prepared, an entire experimental session can be conducted by simply pressing a button when it is time to move to the next step in the planned sequence.

Calibration. Calibration is accomplished by having a boxed dot appear at those positions desired, and having the person fixate the dot directly and press a button. At that time the computer samples the eye position signal and stores the value in a calibration table to be used later in translating from eye monitoring equipment voltage levels to CRT screen locations. A frequent problem in this sort of calibration is that after holding a steady fixation on the target, subjects move their eyes before pressing the button. This is greatly reduced by leaving the dot in place for some time (at least $500 \mathrm{msec}$ ) after the button has been pressed. Another problem is that if the intensity of the ambient lighting is very low, the difference in pupil size produced by having different numbers of characters displayed on the CRT (hence changing the amount of light entering the eye) can change the eye position signal, even when the eye has not moved. This can invalidate calibration information. We have eliminated this problem by raising the level of ambient lighting to minimize pupil size changes from this cause.

The number and location of calibration points is variable and can be specified for a given study.

In conducting a study, the subject is typically asked to complete the calibration task both prior to and following the reading of a passage. If the values obtained differ sufficiently, the data from that passage are discarded.

Data collection. The data collection program is designed to sample the eye position at times synchronized with the clock in the eye movement monitoring equipment as a means of reducing noise in the signal. Data sampling can occur at rates up to 2,000 times/sec, although we have typically taken data points once each millisecond. Either one or two A/D channels can 
be sampled, corresponding to horizontal and vertical eye position. As the data are being taken, the computer identifies the beginning and end of each saccade. This is done by comparing each new data point with the point taken $4 \mathrm{msec}$ earlier. When this difference rises above a settable threshold (typically, a value corresponding to about $4 \mathrm{~min}$ of visual angle), a saccade is declared to have begun. This reliably identifies that a saccade has begun within $34 \mathrm{msec}$ of its actual initiation and is capable of detecting saccades carrying the eye no more than about $1 / 2$ letter position $(10 \mathrm{~min}$ of arc). The termination of the saccade is similarly identified as the point at which a comparable difference value falls below a settable threshold.

Each sample of the eye position yields one or two 12-bit data values. Since the PDP-11 computer uses 16-bit words, 4 bits are unused in each data word. The program sets bit patterns into these bits to mark the data when particular events occur. The beginning and end of each saccade are so marked.

Following the termination of a saccade, the program does not look for the beginning of another saccade for a settable period of time, usually about $20 \mathrm{msec}$. In this way, the eye movement signal changes that take place during the "overshoot" period are not identified as saccades.

On each millisecond the interrupt routine that samples the data also checks to see if a button has been pressed. If so, it sets another bit in the data word to mark the data point appropriately and initiates the appropriate action, depending on which button was pressed. A buttonpress may call a new page of text, initiate a display change of some sort, or simply cause the data to be marked so reaction times can later be obtained. In addition, when display changes are made, one data word is marked when the new pattern is called for and another when the new pattern has been written on the CRT, so that the data can later be examined to see just when a display change was initiated and when it was completed.

At present, all the data are stored on the disk for later examination and reduction. We anticipate moving to on-line reduction during data collection when we are sufficiently sure of the appropriate algorithms to use.

Display control system. This system is designed to handle either alphanumeric or other graphics displays, although the latter is not our present focus. The studies presently underway that involve display changes are being conducted with single-line text displays, in order to obtain the highest accuracy and reliability in eye movement monitoring using only horizontal eye movement data. Pressing a button brings the next line of text on within a few milliseconds, so reading can proceed quite easily, within the constraint that one cannot visually jump from one line to another and return. As we develop vertical eye movement monitoring capability, we anticipate moving to multiline text displays.
When a new line of text is called for, the program expects to receive, first, a line containing a parameter list, indicating on which fixations or saccades, and how long after their initiation (in milliseconds), the display changes are to occur, and, in some cases, the characteristics of the change to be made. The following lines are then display alternatives. If the change is a simple one, one line is simply replaced with the next in this list whenever a target event or time is reached. At the beginning of a fixation or saccade, the list may be initialized, returning to the display of the first line in the list once again. If the change is a more complex one, the display line is constructed by assembling specified parts of the text line alternatives.

The overall program is written to make it easy to produce "display modules," or instructions inserted into EYECON to cause it to produce display changes of various types. Each display module defines the condition under which a display change will occur and the nature of the change. It recieves the parameters preceding each group of lines in the display file and uses them in producing the correct display change at the correct time. At the present time, four display modules exist which produce display changes. The first simply displays the next line in the line group when a prespecified saccade is encountered (say, the fourth forward saccade on the line). If a display change is called for and the last line of a group is being displayed, it is replaced by the first in the group and the cycle begins again. The second display module makes changes at different times (in milliseconds) following the initiation of either the saccade or the fixation. Provision is made for up to nine display changes to occur after different intervals during a single fixation or saccade while reading. This display mode is particularly useful for studies investigating the effects of masking and other display changes during fixations. The third display module drifts the text steadily and at a prespecified rate across the CRT as the person is reading it. The fourth display module identifies the location of the eye at the beginning of each fixation and creates a display having the characters from one line alternative in a particular region specified with respect to the eye position and having characters from the other line alternatives in other regions. A fifth display module, which does not permit eye movement contingent display changes, displays a given number of lines (a "page") on the CRT and monitors both vertical and horizontal components of the reader's eye movements. A new page is called up with each buttonpress. A program has also been written to aid in the development of new display modules, so they can be produced with relative ease.

\section{Characteristics of the System as a Whole}

The signal is quite clean. Noise level is $\pm 2 \mathrm{~A} / \mathrm{D}$ values, where 45 values corresponds roughly to $20 \mathrm{~min}$ of arc, or 1 letter position. Repeatability is also reasonably 
good on the horizontal dimension with careful refixations of the same point by experienced subjects. Vertical repeatability is much poorer. The eye movement monitor has a propagation lag of $2-3 \mathrm{msec}$. As mentioned earlier, the display system requires $2.05 \mathrm{msec}$ to write 71 characters on the screen, using the hardware character generator. There is provision for software generation of nonstandard characters, but this is substantially slower.

Figure 6 shows the speed with which a display change ran be made. This shows one saccade, with a mark at the point where the velocity crossed a threshold set high enough to insure that the saccade would travel 3 letter positions (1 deg of visual angle) or more. At that point the processing required to replace the line being displayed with a new line was initiated. When the display processor completed writing the new line on the screen, the data were marked again. The entire process required only (actually less than) $3 \mathrm{msec}$. The change was completed well before the eye stopped for the next fixation.

Characteristics yet to be implemented. In addition to plans to move toward on-line data reduction and more complete two-dimensional eye tracking, which have already been mentioned, several other improvements are planned. We will add a prediction algorithm to the program, by which the computer can predict during a saccade where and when the next fixation will begin, thus allowing display changes to be made contingent upon this information. A procedure is being prepared for making available to a display module all of the information that might be used in deciding whether a display should be initiated, in a form that makes it easy to define the conditions under which a change should take place. This will include the ability to make the change contingent upon a complex eye movement history; for instance, making a change only during a regression of not more than 3 letter positions which is immediately preceded by at least two

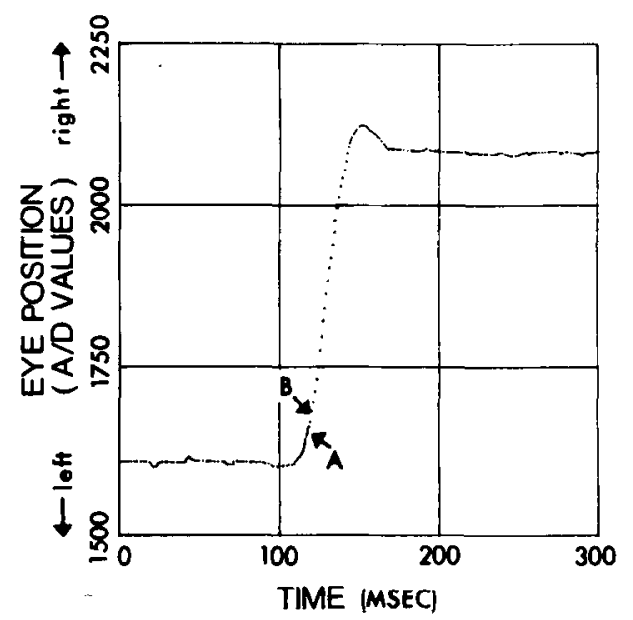

Figure 6. Time required for a display change to be made. The data point marked $A$ is the point at which the computer initiated a display change; at the point marked $B, 3$ msec later, the change was realized on the CRT. consecutive forward saccades of at least 6 letter positions in length. In this way it will be possible to investigate the perceptual and cognitive processes occurring during very specific fixations and saccades.

Finally, we anticipate adding the capability of making display changes in line drawings displayed on the CRT, primarily by replacing one subpicture with another, thus changing a specific part of the image during a saccade.

Data reduction. Data reduction occurs in several steps following collection of the data. First, the program remarks the locations of the beginnings and ends of saccades using a more accurate algorithm, which takes into account data following, as well as preceding, the critical points. This algorithm first locates a saccade on the basis of velocity of eye movement. It then looks leftward toward the beginning of the saccade to find the point at which the movement trend is broken: This is taken as the beginning of the saccade. Finally, it looks rightward toward the end of the saccade to find the point at which movement in the direction of the saccade falls below a threshold. In this way, overshoot movement in the opposite direction is not taken as part of the saccade. Slow drifts (probably vergence movements), which occasionally occur at the ends of long saccades in the same direction as the saccadic movement, are not taken as extensions of the saccade. This algorithm also watches for very short fixations, which are typically indicators of blinks. Such regions are indicated by "ignore data" marks, so that the data will not be considered later. The regions of blinks are then displayed on the CRT and visually examined with occasional editing by inserting and deleting marks through keyboard instructions. The data are then summarized in a reduced form which saves, for each saccade-fixation pair, the following values: duration of the saccade (in milliseconds), maximum saccade velocity, the point at which this was reached, location of the eye at the time the saccade was completed, location of the eye halfway through the fixation (which is taken as the fixation location), duration of the fixation (in milliseconds), location of the eye at the end of the saccade, and elapsed reading time. Finally, various programs operate on the reduced data files to summarize the eye movement characteristics needed for a particular study: distributions of fixation durations, saccade lengths, and so on, figures showing where on the line of text the eye stopped and for how long, and the durations of fixations in specific regions of the text. When information is desired concerning where the eye was centered for a fixation, the calibration data are used, with a simple linear interpolation between the points. A more complex linear translation algorithm is used when two-dimensional translation is required.

Alternative approaches. The program as it is now written, when sampling eye position every millisecond, storing all the data, checking each sample for saccade beginnings or endings, and maintaining some record 
of eye movement patterns for display change decisions, takes approximately $60 \%$ of the CPU time. This leaves time available for the additional computation required for planned enhancements of the program. Much of the time being used is associated with storing the data, so CPU time could be recovered by reducing the sampling rate, or perhaps by on-line data reduction, thus reducing the amount of data to be stored. This, however, suggests that attempts to provide the level of research capability provided in this system on computers much less powerful than the PDP-11/40 may encounter timing difficulties.

It may be possible to write programs for this type of research in FORTRAN if a number of assembly language routines are included to handle the most frequently repeated activities. Care must be exercised or timing difficulties may be encountered.

Another possible approach to developing a system for conducting this type of research is to distribute processing functions among microprocessors. However, this approach is typically most promising in situations where different aspects of the task can be handled in parallel. The task of monitoring eye movements and making display changes based on them is primarily a serial task requiring taking a sample, carrying out certain checks on the value(s) obtained, and then, if needed, doing those things that are required to produce a display change, and at some point storing the data. The primary limitations are on the speed of the CPU and mass storage devices; having parallel processing capability is not particularly helpful.

While this discussion of stimulus control has been in terms of visual displays, it could as easily be used with auditory or other stimulus forms. Thus, tones can be sounded, words presented auditorially, or regions of the brain stimulated electrically when certain areas of the text or picture are fixated, using a very similar technology. In setting up such systems, many of the problems would be similar to those discussed above.

\section{CONCLUSION}

The technology described in this paper opens the door to eye movement based stimulus control in such normal visual tasks as reading, searching through text, or examining a simple picture or map. It is now possible to make stimulus changes contingent upon whether the eye is moving or still, on where the eye is directed, on how long it has been at this location, and so on, while the subject is engaged in the larger, ongoing task. This is an important step in the development of a research capability for studying these complex tasks, and future technological developments will amplify this capability. However, it should be noted that there is a second step that is equally important for such research. This is the development of appropriate dependent variables. While it is now possible to inject highly specific stimulus manipulations into an ongoing task, we need equally refined methods of assessing the effects of these manipulations without changing the subject's task. Eye movement records provide a relatively unobtrusive measure of behavior, but much more research is needed to specify the relation between the eye movements and the cognitive and perceptual processes before such data can be effectively used (McConkie, Note 5). The fact that specific aspects of the eye movement patterns have been affected by the stimulus manipulations produced by McConkie and Rayner (1975) and by Rayner (1975) gives some hope that such data will indeed serve as an effective measure, but much work still needs to be done on this problem.

\section{REFERENCE NOTES}

1. McConkie, G. W., \& Rayner, K. Identifying the span of the effective stimulus in reading. Final report, U.S. Office of Education Grant OEG-2-71-0531, July 1974.

2. McConkie, G. W., \& Zola, D. Is visual information integrated across successive fixations in reading? Manuscript in preparation.

3. McConkie, G. W., \& Zola, D. How precise is eye guidance in reading? Manuscript in preparation.

4. Levy-Schoen, A., \& O'Regan, K. The control of eye movements in reading. Paper presented at the Conference on Processing of Visible Language, Eindhoven, The Netherlands, September 1977.

5. McConkie, G. W. On the role and control of eye movements in reading. Paper presented at the Conference on Processing of Visible Language, Eindhoven, The Netherlands, September 1977.

\section{REFERENCES}

Al.PERN, M. Effector mechanisms in vision. In J. W. Kling \& L. A. Riggs (Eds.), Experimental psychology (3rd ed.). New York: Holt, Rinehart, \& Winston, 1971.

Cornsweet, T. N., \& Crane, H. D. Accurate 2-dimensional eye-tracker using first and fourth Purkinje images. Journal of the Optical Society of America, 1973, 63, 921-928.

Forster, K. I. Visual perception of rapidly presented word sequences of varying complexity. Perception \& Psychophysics, 1970. 8. 215-221.

Matin, E. Saccadic suppression. Psychological Bulletin, 1974, 81, 899-917.

McConkie, G. W., \& RAyner, K. The span of the effective stimulus during a fixation in reading. Perception \& Psychophysics, 1975, 17, 578-586.

McConke, G. W., \& RAYneR, K. Asymmetry of the perceptual span in reading. Bulletin of the Psychonomic Society, $1976,8,365-368$.

O'REGAN, J. K. Structural and contextual constraints on eye movements in reading. Unpublished doctoral dissertation, University of Cambridge, 1975.

PotTER, M. Short-term conceptual memory for pictures. Journal of Experimental Psychology: Human Learning and Memory, 1976, 2, 509-522.

RAYNER, $K$. The perceptual span and peripheral cues in reading. Cognitive Psychology, 1975, 7, 65-81.

REDER, S. M. On-line monitoring of eye position signals in contingent and noncontingent paradigms. Behavior Research Methods \& Instrumentation, 1973, 5, 218-228.

Taylor, G. A., Klitzke, D., \& Massaro, D. A visual display system for reading and visual perception research. Behavior Research Methods \& Instrumentation, 1978, 10, 148-153.

Young, L. R., \& Shenna, D. Survey of eye movement recording methods. Behavior Research Methods \& Instrumentation, 1975, 7, 394-429. 